\title{
Former à la méthodologie de la recherche en Sciences Humaines et Sociales : le cas des masters Universitaires Français
}

\author{
Training in the methodology of research in the Humanities \\ and Social Sciences: the case of the French University \\ Masters
}

Benoît Raveleau*

\section{Résumé}

La réalisation d'un mémoire de recherche constitue une pratique pédagogique commune à la plupart des masters de sciences humaines et sociales des universités françaises. Pour préparer les étudiants à ce travail, un enseignement à la méthodologie de recherche et un accompagnement individuel sont généralement proposés. Cet article identifie les principales difficultés rencontrées autant pas les étudiants que par les professeurs qui les accompagnent dans ce travail. Cinq modèles de formation à la méthodologie de recherche sont distingués, identifiés et discutés afin d'optimiser les pratiques de direction de

PBR : Docteur en sociologie du travail, e-mail : benoit.raveleau@uco.fr 
mémoire. Nous concluons sur quatre défis éducatifs auxquels sont confrontés les conseillers de recherche, par-delà les disciplines.

Mots-clés : Formation. Méthodologie. Recherche. Master.

\section{Resumen}

The production of a research dissertation constitutes a pedagogical practice common to most of the masters of humanities and social sciences of the French universities. In order to prepare the students for this work, teaching in research methodology and individual coaching are generally proposed. This article identifies the main difficulties encountered not only by the students but also by the professors who accompany them in this work. Five training models in the research methodology are distinguished, identified and discussed in order to optimize memory management practices. We conclude on four educational challenges faced by research advisors, across disciplines.

Keywords: Training. Methodology. Research. Master.

\section{Introduction}

La réalisation d'un mémoire de recherche constitue une pratique pédagogique commune à la plupart des masters de sciences humaines et sociales des universités françaises. La justification apportée à cette activité va bien au-delà des enjeux liés à l'acquisition de compétences de recherche, car très peu des étudiants concernés se destinent à devenir chercheur professionnel. La très grande majorité exercera dans des professions de l'humain (enseignement, éducation, métiers du social, du socio-culturel, de la communication, de l'accompagnement des personnes, etc.). Le travail de recherche demandé aux étudiants de master est donc le plus souvent appréhendé comme outil 
de professionnalisation dans lequel l'écriture est à la fois moyen et médium, aussi bien pour la communication que pour l'élaboration et le développement de la pensée.

Pour réaliser ce mémoire, les étudiants se voient le plus souvent proposer un enseignement de méthodologie de recherche et un accompagnement individuel grâce à l'appui d'un directeur de recherche, enseignant-chercheur de la discipline concernée. Cet article a pour but de discuter des méthodes utilisées pour former à la méthodologie de recherche et de confronter les pratiques de direction de mémoire afin de les optimiser. Il s'appuie sur l'expérience de formation et d'accompagnement d'une vingtaine d'années de l'auteur auprès d'étudiants de master et doctorat en Sciences humaines et sociales. Cette synthèse permet de dégager quelques tendances communes, même si elle ne prétend pas dresser un tableau exhaustif des pratiques de formation à et par la recherche dans les universités françaises.

Dans cet article, nous retiendrons uniquement trois axes principaux : les enjeu d'une formation par la recherche en master universitaire, les modèles de formation utilisés pour développer les compétences de recherche des étudiants, les principales difficultés rentrées par les étudiants. Nous concluons sur quatre défis auxquels sont confrontés les conseillers de recherche, par-delà les disciplines, en ouvrant des perspectives pour des recherches à venir.

\section{Considérations générales sur la place de la recherche en master universitaire}

Depuis les années 1970 et l'essor des effectifs universitaires en France, acquérir les connaissances de méthodologie de la recherche nécessaires à la réalisation d'un mémoire est un objectif pédagogique que proposent la plupart des Masters en sciences humaines et sociales. La justification souvent apportée à la prolifération de ce type d'enseignements, insiste sur le fait que la recherche constitue l'un des attributs essentiels 
de l'Université. De fait, on peut résumer les fonctions de cette dernière en trois missions caractéristiques :

- Transmettre des connaissances et savoir-faire (Enseignement).

- Produire des connaissances nouvelles (Recherche).

- Rendre des services à la communauté environnante (Intervention)

Si l'universitaire quelle que soit sa discipline essaie à travers maints tâtonnements d'établir pour lui-même un équilibre entre ces trois missions, il n'est est pas du tout de même pour l'étudiant. La force des traditions universitaires confine ce dernier pendant ses premières années passées dans l'enseignement supérieur à la première mission. Il consacre tout son temps à la réception des enseignements et retransmission au cours d'exposés, de séminaires, de travaux de groupes, de différentes épreuves écrites ou orales.

Et c'est souvent brutalement au bout de plusieurs années de travail universitaire que cet étudiant découvre subitement les deux autres fonctions de l'Université : en préparant un mémoire de master, à plus forte raison une thèse de doctorat, il est subitement placé devant cette nécessité de produire de nouvelles connaissances, des connaissances jusque-là inédites. Pour cela, il doit faire œuvre de création intellectuelle. De même, la découverte du milieu professionnelle à la faveur d'un stage long lui fait prendre conscience que non seulement il doit savoir, mais il doit aussi se rendre utile, en s'insérant dans un environnement.

Cette découverte de ce qu'est l'Université se fait donc tardivement et souvent de façon frustrante. D'une certaine façon, on peut considérer qu'actuellement la véritable entrée à l'université se situe en master. Elle doit s'accompagner d'une aide effective pour que l'étudiant apprenne à comprendre et dominer les nouvelles tâches qui lui sont demandées. C'est le sens de l'enseignement à la méthodologie de recherche. Ce dernier, en se centrant sur la deuxième mission de l'Université n'exclue pas 
les deux autres : les trois missions sont étroitement liées et vouloir les disjoindre peut paraître très préjudiciable. On prendra le simple exemple d'un mémoire de recherche. Ce dernier, s'il veut prouver sa qualité, ne doit-il pas chercher à articuler à sa façon :

- La transmission des connaissances déjà acquises sur laquelle doit prendre appui la présente recherche.

- L'élaboration d'un nouveau corps d'hypothèses soumises à validation.

- La connaissance d'un certain terrain professionnel, ou une situation concrète, terrain et situation vis-à-vis desquels sont proposés de nouveaux outils de compréhension.

D'ailleurs, au cours de son cursus universitaire, l'étudiant sera amené à se frotter à cette triple articulation à travers trois types de production caractéristiques. Comme le montre le tableau ci-dessous, chacune de ces productions entend valoriser l'une des trois missions évoquées ${ }^{1}$.

Tableau 1 - Types de production en fonction des trois missions de l'Université

\begin{tabular}{lll}
\hline Missions & Productions & Fonctions \\
\hline $\begin{array}{l}\text { Transmission des } \\
\text { connaissances }\end{array}$ & Fiche de lecture, note critique & Epistémique \\
\hline $\begin{array}{l}\text { Production des } \\
\text { connaissances }\end{array}$ & Mémoire de recherche & Heuristique \\
\hline Service à la communauté & Mémoire d'analyse de la pratique & Analytique \\
\hline
\end{tabular}

1 Dans «La philosophie du Non ». Paris: PUF, Gaston Bachelard affirme de façon très opportune que « penser scientifiquement, c'est se placer dans le champ épistémologique intermédiaire entre Théorie et Pratique ». 


\section{Qu'est-ce que la méthodologie de la recherche ?}

Les étudiants en SHS sont parfois rebutés par l'expression «méthodologie de la recherche ». Le langage et les outils apparaissent réservés à un cartel d'initiés. En fait, il ne s'agit pas ici de faire des découvertes scientifiques, mais d'éviter, en réfléchissant sur les méthodes de travail, de s'éparpiller, perdre son temps, faute d'avoir réfléchi. Avant d'aborder la question de la formation des étudiants à la méthodologie de recherche, il est utile de préciser succinctement les deux principaux termes utilisés : recherche et méthodologie.

\section{Comment définir une recherche?}

Pour B. Gauthier (2009), la recherche peut être définie comme " une activité de quête objective de connaissances sur des questions factuelles » qui se distinguent des connaissances de sens commun. De ce point de vue, une recherche est toujours un accroissement de connaissances. L'université est en fait un lieu où l'on construit des connaissances et où on les transmet, cependant ces connaissances n'ont pas toujours le même statut, la même portée, les mêmes intentions. Selon Angers (2000 [1996] :9), on peut caractériser les recherches SHS en fonction de certains paramètres qui y président. On retiendra ici les trois critères principaux - à savoir, l'intention du chercheur, la visée de la recherche et le mode de prélèvement. Le critère lié à l'intention du chercheur permet par exemple de cerner l'opposition entre recherche fondamentale et recherche appliquée. Le critère de la visée permet de spécifier davantage l'intention du locuteur en spécifiant les buts scientifiques que le chercheur s'assigne (visée descriptive, visée classificatrice, visée explicative, visée compréhensive, visée contrastive). Le critère de prélèvement permet d'opposer les recherches qualitatives aux recherches quantitatives.

Les manuels de recherche en sciences humaines et sociales présentent le plus souvent la démarche hypothético-déductive comme 
la matrice de toutes les recherches (Quivy \& Van Campenhoudt, 1991). Selon les canons établis de cette orthodoxie académique, une recherche doit répondre à 3 critères bien repérés par A. Weinberg (1991) :

- La recherche porte sur un objet limité : Qu'il s'agisse pour le sociologue d'étudier les facteurs qui influencent le vote communiste à Cuba, pour l'historien de comprendre par quelles voies les Celtes sont entrés en Bourgogne ou pour le psychologue de déterminer quel peut être l'effet du bilinguisme sur l'intelligence d'un groupe d'enfant, le travail de recherche porte en général sur un sujet limité et parfaitement circonscrit.

- La recherche vise à répondre à une question précise : "Toute connaissance est la réponse à une question ", disait Gaston Bachelard. Une recherche n'est pas simplement un travail empirique de recueil de données, elle doit répondre à une question, apporter des éléments d'interprétation et d'explication. Si la question est " quelles sont les causes diplomatiques de la participation des ÉtatsUnis à la Seconde Guerre mondiale ? ", il ne s'agit pas de proposer une théorie générale anthropo-socio-historique de la guerre... Les jeunes thésards apprennent vite que plus la question est « pointue ", plus la réponse est humble, plus le directeur de recherche sera satisfait.

- La recherche suit une démarche démonstrative : La production des connaissances en sciences humaines et sociales est marquée par une quête constante des critères de scientificité. Par démonstration, les sciences de la nature comme les sciences humaines ne se prêtent que rarement à des déductions aussi rigoureuses qu'en mathématiques ou sciences physiques. Mais un ensemble de conditions comme l'utilisation et l'intégration du maximum de faits pertinents connus, la logique de la déduction, la précision des concepts, l'absence de jugements de valeurs et de postulats non explicités font partie des règles minimum de la communauté scientifique. 


\section{La méthodologie de recherche comme démarche itérative}

Toute méthodologie, y compris donc la méthodologie de la recherche, est à entendre à double sens : comme une démarche, comme une réflexion sur cette démarche. La démarche n'est pas forcément à lire de façon linéaire et systématique comme nous le verrons plus loin. La démarche n'a de valeur qu'en fonction de la valeur de son maillon le plus faible. C'est dire que toute démarche est tributaire d'un petit démon très mobile qui tantôt se logera au niveau de la détermination de l'objet, tantôt au niveau des méthodes choisies, tantôt au niveau de la population retenue, tantôt au niveau du recueil ou du traitement des données. La réflexion implique quant à elle un retour sur les démarches antérieures, même si ces démarches avaient moins d'ampleur que la démarche actuelle. Une telle réflexion doit déjà pouvoir fournir tout un savoir méthodologique critique. À cette première réflexion sera joint un regard critique sur la démarche méthodologique menée actuellement. La réflexion sur la démarche n'attend pas le terme de celle-ci. Elle se fait à intervalles réguliers au cours de son déroulement pour mieux en saisir les enjeux.

\section{La méthodologie c'est également la combinaison de 2 processus d'interaction (démarche itérative) :}

- La pratique, par le biais de l'observation des situations réelles, des situations de travail. C'est le domaine du faire, de l'expérience, de l'observation d'un objet. Ce qu'écrivait déjà dès 1934, Kurt Goldstein : "Méthode comme théorie ne doivent prendre naissance qu'à partir de ce qu'il y a de plus concret » (p. 433). Pour explorer des situations concrètes et dépasser le stade de la simple perception, il sera utile par exemple de mettre en place des outils de recueils de données (entretien, questionnaires, analyse de documents, etc.). Ces outils seront choisis en 
fonction de leur pertinence avec le thème étudié. Par exemple, pour des recherches réalisées dans le secteur de la santé :

- Appréhender des problèmes relationnels ou émotionnels comme la souffrance des soignants vis-à-vis de la mort avec des entretiens.

- Appréhender des problèmes comportementaux comme des gestes d'hygiène dans un service avec des grilles d'observation.

- Étudier la tenue des dossiers de soins à l'aide d'une analyse de contenu thématique.

- Étudier l'opinion des infirmières sur la dernière réforme hospitalière avec des questionnaires.

- La réflexion par l'intermédiaire des savoirs mobilisés. La théorie consiste à donner un sens à la pratique ; elle renvoie aux domaines des savoirs, des principes. Pour conduire tout travail méthodologique, il est nécessaire de se référer à des cadres de pensées scientifiques, qui sont utiles pour lire la pratique et lui donner sens. Cette étape peut s'avérer délicate pour des étudiants de Master qui ne disposent pas toujours des connaissances théoriques suffisantes. À leur décharge, reconnaissons que les cadres théoriques sont multiples dans les sciences humaines et sociales. Plus précisément, la problématique peut être tributaire de plusieurs niveaux d'appréhension :

- Les références idéologiques, philosophiques, les finalités poursuivies par le chercheur : on insistera alors sur une problématique égalitariste, libertaire, chrétienne (voire thomiste), fixiste, innéiste, etc.

- Les théories choisies : on évoquera une problématique fonctionnaliste, psychanalytique, cognitiviste, structuraliste, interactionniste, etc. 
- La discipline valorisée. Cela peut être aussi bien des références empruntées aux sciences de l'éducation, à la psychologie, à la psychologie sociale, sociologie, ergonomie, économie, etc.

Cette approche multi référentielle est souhaitable, car elle enrichit la compréhension des situations : Par exemple, si vous souhaitez étudier le problème de l'absentéisme dans l'industrie agro-alimentaire, plusieurs possibilités s'offrent au chercheur :

- Choisir un modèle sociologique qui amène à réfléchir sur le travail des femmes dans la société (les équipes de travail de ce secteur étant essentiellement féminines).

- Choisir une approche psychosociologique qui amène à prendre en compte les relations de travail au sein des équipes féminines de travail.

- Choisir une approche ergonomique qui va faire référence aux conditions de travail et d'hygiène.

Le choix du modèle utilisé pour lire la pratique va donner une collaboration très particulière à la situation observée. Il est donc important d'être au clair avec le modèle, de vérifier sa pertinence avec l'objet du travail et de construire des outils de recueil de données cohérents avec ce modèle.

Lorsqu'on la présente aux étudiants comme essentiellement constituée d'étapes successives, ou d'outils et de procédures, la méthodologie devient un simple gadget qui occulte la finalité du travail. Or, la création intellectuelle à l'œuvre dans la recherche est souvent le fruit d'une longue incubation faite d'expérience entrecoupée de lectures et de réflexions tant sur les choses que sur soi-même. C'est dire que la construction de l'objectivité est capricieuse et peut se définir à travers l'exercice permanent de l'esprit critique. De ce fait, elle ne renvoie pas à un état final susceptible d'être atteint. Elle requiert plutôt une vigilance permanente, 
en particulier lors des différentes phases mentionnées ci-dessous à l'articulation des aspects théoriques et pratiques (Problématisation de la recherche, opérationnalisation du cadre d'analyse, interprétation des données, Recommandation suite aux résultats).

Figure 1 - La recherche comme démarche itérative entre pratique et théorie

Théorie

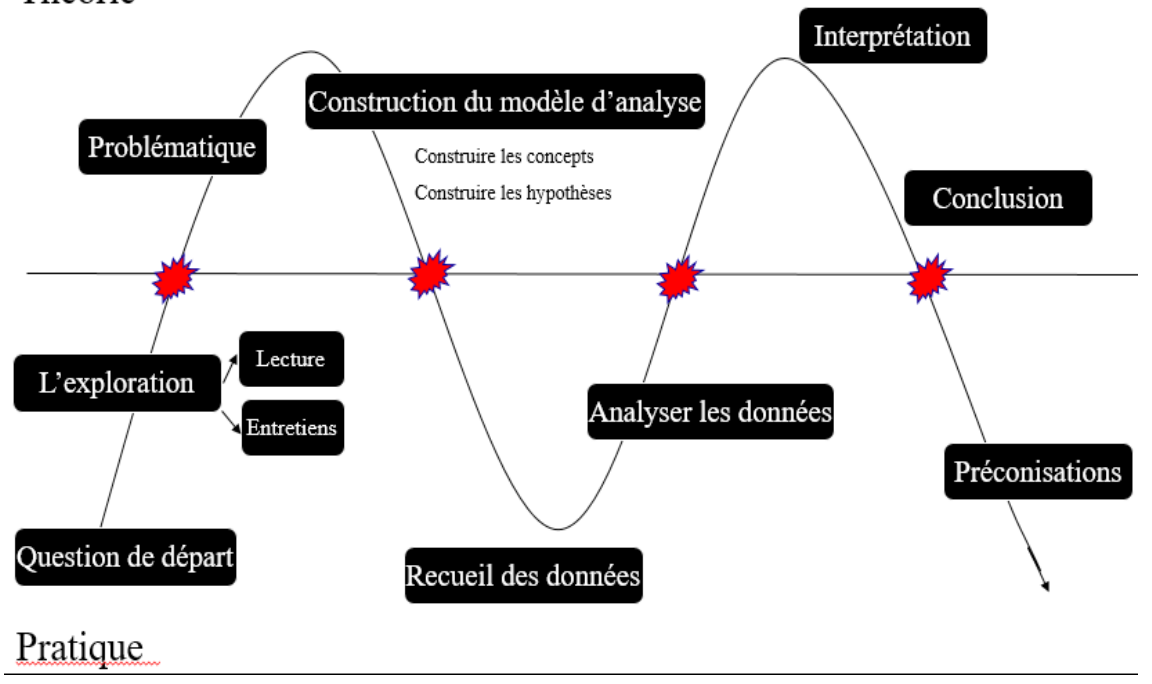

\section{Enjeux d'une formation à la recherche dans les SHS ?}

Une réflexion sur la formation à la recherche est indissociable d'une analyse des enjeux de toutes les activités destinées à produire du savoir. Pourquoi est-il utile de développer des compétences relatives à la recherche pour des étudiants qui ne se destinent pas à un parcours académique, mais souhaitent exercer comme psychologue en entreprise, enseignants en collège ou encore chargés de communication. Autrement dit, comment motiver des étudiants à s'investir dans la réalisation d'un 
travail de recherche qui peut parfois apparaître comme éloigné des réalités professionnelles. Pour les sensibiliser aux enjeux présents dans ce type d'enseignement, il importe sans doute de rappeler la distinction devenue classique entre formation PAR la recherche et formation POUR la recherche. Sur ce point, on consultera ici avec intérêt le numéro spécial de la revue Recherche et formation publié en 2008 et intitulé "Formation à la recherche, formation par la recherche ».

- La formation POUR la recherche est présente dans des formations de types Master recherche ou Doctorat. Généralement, un parcours de formation à la recherche est proposé aux étudiants de Master dès la première année de master. Il permet à l'étudiant(e) de développer ses connaissances et ses compétences en recherche. Il l'oriente vers la recherche appliquée et fondamentale dans ce domaine. Il lui offre notamment la possibilité d'acquérir un niveau scientifique requis pour faciliter l'accès aux métiers de la recherche.

- Formation PAR la recherche dans les formations professionnalisantes de type Master professionnel ou École professionnelle. Dans cette perspective, il convient de lever certaines idées reçues, voire certains préjugés, en soulignant que la professionnalisation est un processus à interroger au-delà de la seule sphère technique de l'apprentissage d'un métier. Se former par la recherche, c'est aussi mieux comprendre les fonctionnements professionnels et apporter une véritable plus-value au monde de l'entreprise. La recherche permet par exemple d'acquérir des savoirs, d'apprendre à trouver et traiter une information, d'apprendre des méthodes de résolution de problème, de savoir communiquer à l'oral ou par écrit.

Bien sûr, comme le souligne Jean-Marie Van der Maren (2008), le souci de "vérité » et donc les critères d'évaluation de la recherche ne sont pas les mêmes dans l'ordre de la recherche scientifique et dans l'ordre 
de la recherche quotidienne. Le futur professionnel est moins enclin à rechercher la vérité que l'efficacité de son action. On comprend alors mieux la relative indifférence des étudiants de l'Université aux questions d'épistémologie et de méthode. C'est dans cet esprit que les responsables de formations universitaires, chargés d'organiser les enseignements, visent à tenir simultanément la volonté d'accompagner la finalité d'insertion professionnelle et la volonté d'accompagner l'autonomie intellectuelle, mais dans une double injonction relativement classique : les logiques de fonctionnement, la temporalité et les attentes ne sont pas les mêmes dans une entreprise et dans la formation à et par la recherche. La valorisation de la connaissance doit être immédiate dans l'entreprise, en termes d'innovations par exemple, et la valorisation de la connaissance dans la recherche demande un effort de réflexivité qui n'est pas immédiatement opérationnalisable. C'est tout l'enjeu de la professionnalisation des acteurs et des structures.

Pour les étudiants de Master SHS, au moins 4 enjeux caractérisent cet enseignement de méthodologie de la recherche :

- L'enjeu personnel pour l'apprenant se situe dans l'extension de leurs connaissances, dans la découverte de leurs capacités intellectuelles et des qualités morales associées à tout travail intellectuel (humilité, tolérance, prudence, curiosité, etc.). Sous des appellations variées, la plupart des conceptions actuelles de la motivation partagent l'idée selon laquelle la réussite et la confiance en nos capacités à agir efficacement jouent un rôle crucial dans notre engagement et nos performances (BANDURA, 1997).

- L'enjeu professionnel se situe principalement dans l'espoir d'un meilleur statut, d'une mobilité plus grande, que ce soit pour les étudiants en voie directe ou pour ceux en reprise d'étude. Le travail de recherche constituant souvent la pièce maîtresse de la validation de nombreux masters, sa bonne évaluation peut constituer un point de départ dans la reconnaissance professionnelle. 
Il peut permettre à l'étudiant d'approfondir une expertise professionnelle et de se faire reconnaître auprès de ses pairs.

- L'enjeu social c'est la possibilité pour eux et leur groupe professionnel de poser un diagnostic social. De ce point de vue, la recherche en sciences humaines et sociales n'est pas seulement l'affaire des spécialistes. Elle concerne un grand nombre de personnes (travailleurs sociaux, professionnels des secteurs culturels, sanitaires, éducatifs, psychologiques, etc.). La mise en place en France depuis 25 ans de Masters à finalité professionnelle qui comprennent la réalisation d'un mémoire de recherche peut aussi être lue comme une volonté d'autonomisation des professionnels par rapport aux champs scientifiques (médical, psychologique, etc.) et par rapport aux décideurs (politique et administration de tutelle). Certains praticiens réflexifs inscrits en Master tentent de la sorte de parfaire leur professionnalisation en se constituant un objet d'étude autonome, voire comme discipline à part entière.

- L'enjeu communicationnel : La production d'un savoir propre aux groupes professionnels sert tout autant à la communication dans le groupe qu'à l'extérieur du groupe. Dans le groupe, cette communication permet la production d'un savoir commun, d'un discours commun et par-delà d'une représentation commune de la réalité. La recherche en milieu professionnel joue de ce point de vue le rôle d'une société de pensée qui sert à fabriquer une opinion commune. À l'extérieur du groupe, la capacité des professionnels à réaliser des recherches permet une production d'arguments dans l'optique de convaincre et de se convaincre. Pour revendiquer le statut de professionnel et non plus seulement de salarié, la recherche devient un nouveau mode de communication.

Pour autant, la recherche pose des problèmes difficiles aux praticiens qui reprennent des études universitaires dès qu'ils veulent se 
donner les moyens d'analyser scientifiquement des faits en relation avec leur pratique. Les raisons de ces difficultés sont multiples : Problèmes institutionnels et organisationnels pour faire reconnaître cette fonction de recherche dans son activité quotidienne ; Problèmes théoriques et méthodologiques liés à l'absence d'une formation antérieure à la recherche ; Problèmes éthiques de la pratique et de la recherche en milieu professionnel ; Problèmes pratiques de réalisation (temps, argent).

Par ailleurs, le chercheur et le professionnel ne doivent sans doute pas être confondus : Le chercheur est celui dont la fonction principale est la recherche (processus intentionnel, systématique, ajusté et évalué). Le professionnel de terrain est celui dont la fonction est de produire un service en faisant appel à ses connaissances (expertise professionnelle). Même si ces deux groupes d'acteurs ont cependant en commun que l'information constitue une étape initiale permettant de guider leur action.

\section{Comment former les étudiants de SHS a la recherche?}

Il existe de multiples façons de former des étudiants de Master à la recherche. Par exemple, une première voie consiste à les faire prendre contact avec les résultats de la recherche. Lecture d'ouvrages et consultation d'articles scientifiques peuvent fournir des exemples de bonnes pratiques de recherches ou donner des idées de solutions méthodologiques pour le recueil ou l'analyse des données. Autrement dit, une recherche ne naît pas de rien. La recherche ce n'est pas que de la méthodologie. Elle nécessite de disposer de connaissances théoriques et de prendre les moyens de développer sa curiosité intellectuelle, sa capacité d'étonnement devant les gens, les choses, les situations. La curiosité intellectuelle vise autant les productions scientifiques déjà réalisées que l'actualité qui se déroule sous nos yeux. Une seconde voie consiste à former les étudiants au processus de recherche lui-même : comment transformer un problème d'action en un problème de connaissance ? Aider les étudiants à entrer dans la recherche (et sans doute aussi à un autre moment, les aider à en sortir). 
Pour ce faire, la présentation de l'approche hypothético-déductive constitue la méthode de recherche privilégiée dans l'enseignement supérieur (Cf. QUIVY et VAN CAMPENOUGHT, 2011).

Mais la formation des étudiants SHS à la recherche pose quelques problèmes didactiques aux enseignants chargés de conduire des enseignements dans ce domaine. Comment amener les étudiants à se départir d'attitudes professionnelles spontanées quant aux évaluations qu'ils portent sur les faits ? Qu'est-ce qui permet une réelle appropriation des méthodes utilisées? Que peut-on dire sur la programmation des opérations et sur l'interprétation des résultats ? En nous appuyant sur notre expérience d'enseignant universitaire, nous examinerons ci-dessous les apports successifs de cinq modèles qui, quoique différents, sont néanmoins complémentaires dans un processus de formation visant la maîtrise de l'ensemble de la procédure de recherche.

\section{L’approche Épistémologique}

Ce premier modèle offre aux étudiants un ensemble de réflexions sur les conditions objectives nécessaires à la production d'un savoir scientifique. L'accent est mis ici sur la construction de l'objectivité et invite l'apprenti chercheur à avoir une réflexion épistémique à travers laquelle il s'interroge pour savoir en quoi les instruments théoriques ont une validité scientifique, en quoi au contraire ils sont en dehors du champs de la connaissance scientifique. Seul le niveau épistémique pourra dire ce qui relève du savoir scientifique, ce qui s'en écarte de trop. L'épistémologie exerce « une fonction de vigilance critique » en dictant des règles propres à la démarche scientifique et plus précisément celles qui concernent la rupture avec la connaissance vécue et la construction de l'objet scientifique.

Le respect de certains principes épistémologiques fait partie des règles minima de la communauté scientifique. Citons à titre d'illustrations : 
- L'utilisation et l'intégration d'un maximum de faits pertinents connus.

- La précision des concepts, la nécessité d'une définition provisoire de l'objet d'étude.

- L'absence de jugement de valeurs et de postulats non explicités.

- La prudence et l'humilité dans l'interprétation des résultats.

- La tolérance à l'égard des pairs, des autres théories.

- Le combat contre les généralisations hâtives et faciles.

- La conquête du fait scientifique contre l'illusion du savoir immédiat.

- Les pièges de la métaphore, du prophétisme, de l’animisme, du substantialisme, del'anthropomorphisme, del'ethnocentrisme...

\section{Exemple :}

Une étudiante de Master de psychologie envisage d'analyser les facteurs explicatifs de l'augmentation des tentatives de suicide chez les jeunes, phénomène observable selon elle à travers diverses manifestations : reportage dans les médias, personnes de son entourage, rencontre avec des jeunes lors de stage à l'hôpital... La réflexion épistémologique l'amène à se garder de son expérience personnelle et professionnelle ou des généralisations abusives qu'elle serait tentée de faire à partir du cas de quelques jeunes connus d'elle. Ceci peut aller jusqu'à une remise en cause de la validité même de l'objet d'investigation l'augmentation supposée pouvant en partie résulter d'une plus grande focalisation des services sociaux ou des médias sur ce type de population ou d'une moindre tolérance des habitants à l'égard de comportements plus ou moins asociaux.

L'épistémologie forme l'esprit scientifique des étudiants en déterminant chez eux une attitude qui les conduit à considérer autrement les faits qui les entourent et à se défier des évidences du sens commun, mais elle ne règle aucun des problèmes méthodologiques et techniques qu'ils vont rencontrer dans leur démarche de chercheur. Comment constituer mon échantillon ? Quel type d'analyse des données appliqué ? Comment structurer mon mémoire ? etc. C'est pourquoi, quand les apports épistémologiques 
ont révélé leurs limites, les contenus de formation s'orientent souvent vers les aspects techniques relatifs aux instruments utilisés dans les recherches.

\section{L’approche par les techniques}

En sciences humaines, il est aussi possible de former les étudiants aux six techniques de recherche ou moyens d'investigation. On obtient des données par l'une ou l'autre de ces techniques, qui peuvent aussi être regroupées en deux grandes classes selon le type de données qu'elles produisent. Chacune de ces techniques de recherche libère soit des données primaires, soit des données secondaires. Les données primaires sont les nouvelles données obtenues au cours de la recherche, alors que les données secondaires sont celles qui existent déjà. Le tableau suivant inspiré de M. Angers (1996), montre les types de données produites par chacune des six techniques de recherche.

Tableau 2 - Les types de données produites par les six techniques de recherche

\begin{tabular}{l|c|c}
\hline \multirow{2}{*}{ Techniques de recherche } & \multicolumn{2}{|c}{ Données } \\
\cline { 2 - 3 } & Primaires & Secondaires \\
\hline L'observation en situation & $\mathbf{X}$ & \\
\hline L'entrevue de recherche & $\mathbf{X}$ & \\
\hline Le questionnaire ou sondage & $\mathbf{X}$ & \\
\hline L'expérimentation & $\mathbf{X}$ & $\mathbf{X}$ \\
\hline L'analyse de contenu & & $\mathbf{X}$ \\
\hline L'analyse de statistiques & & \\
\hline
\end{tabular}

L'intérêt pour les étudiants est qu'ils y trouvent des procédures précises, codifiées et objectives qui permettent d'avancer concrètement dans le recueil et l'analyse des données. Pour cette raison, ces indications techniques sont, en général, bien reçues par les étudiants, 
même si leur utilisation n'est pas toujours maîtrisée. Trois difficultés au moins sont repérables :

- La concision et le schématisme avec lesquels sont présentés ces aspects techniques entretiennent parfois dans l'esprit des étudiants une illusion de simplicité et un sentiment de surdétermination de leur démarche de recherche. Il suffirait de suivre avec sérieux les différentes opérations techniques à effectuer pour être en mesure de mettre en oeuvre une enquête ou une analyse de contenu. Il s'agit là d'une idée répandue, aisément observable à travers les conduites des étudiants vis-à-vis des questionnaires, par exemple. Pourtant, l'utilisation adéquate et efficace d'une technique est en partie seulement déterminée par le respect de procédures instrumentales. Le reste est une affaire qui engage la capacité de réflexion et d'invention du chercheur.

- Une seconde difficulté, renvoie au risque encouru par les étudiants de s'écarter de la réalité humaine qui a motivé leur recherche, par excès de conformisme aux exigences techniques. Un exemple fréquemment rencontré en formation est celui où des étudiants élaborent et diffusent un questionnaire sans formuler au préalable ce qu'ils en attendent. En fait, le questionnaire vient implicitement justifier une décision, une pratique ou une situation antérieure à sa construction.

\section{Exemple :}

Un établissement de santé prévoit la mise en place d'une structure d'accueil ou de prise en charge. Elle fait procéder, auprès de la population potentiellement concernée, à une enquête de base qui sera indiscutable sur le plan strictement technique : l'échantillon est représentatif, la formulation des questions est peu inductive, le questionnaire est précis et concis, le traitement statistique est multivarié pour permettre d'établir des typologies, etc. Mais si les promoteurs de la recherche en question se limitent à ce seul aspect 
technique, il est probable que les résultats observés correspondront davantage à la perception qu'eux ont des besoins et viendront simplement rationaliser une décision d'implantation acquise implicitement.

On peut penser qu'un instrument de recueil ou de traitement des données peut aussi devenir un refuge rationalisé et confortable. On peut supposer l'existence d'une relation entre la propension à utiliser des outils standardisés et quantitatifs, d'une part, et «l'inconfort » psychologique dans l'activité professionnelle. Par exemple, certains praticiens en reprise d'étude en Master sont pris quotidiennement dans des systèmes professionnels perçus comme instables, insécurisants. Ils manifestent un intérêt quasi exclusif à l'égard de toutes les techniques standardisées, instrumentales, quantitatives. Ils trouvent dans la manipulation des questionnaires ou des données statistiques une sécurité intellectuelle et personnelle loin des aléas et des vicissitudes de leurs activités habituelles auprès des patients. Au terme de leur démarche, ils sont alors comme le savant de Gaston Bachelard (1938), pris dans « une émeute de chiffres », ils croient " au réalisme de la mesure plus qu'à la réalité de l'objet ».

- Un troisième biais introduit par le technicisme est lié à la fonction d'utilité pragmatique. "Trouver une utilité, c'est trouver une raison " écrit Bachelard dans son écrit de 1938 : considérations pragmatiques qui conduisent à envisager l'utilité comme principe d'explication. Les propos de Bachelard ne sont pas éloignés des questions afférentes à la formation à la recherche appliquée. En effet, le rationalisme empirique est la méthode spontanée des étudiants en SHS qui ont la volonté de rompre avec la connaissance sensible, intuitive, vécue.

Une quantité importante d'observations, d'informations, de notes, d'indications diverses sont amassées, elles semblent aux 
étudiants toutes utiles parce que se rapportant à la période ou à l'espace considéré, mais elles sont difficilement traitables d'un point de vue analytique. Ce principe général d'intérêt « tout est important " et d'utilité « tout peut servir par la suite » condamne à un tri a posteriori dans le matériau recueilli. Le refus de se doter a priori de critères de sélection pour la collecte de données rend l'explication et l'analyse délicates sinon impossibles. Les étudiants ne sont plus en capacité de déterminer dans la masse d'informations ce qui est important au regard des objectifs initiaux, ce que seul un corps d'hypothèses structurées peut permettre.

\section{Le modèle linéaire}

Les difficultés rencontrées à partir d'apports trop strictement limités à l'épistémologie ou à la technologie ont induit une présentation de la recherche comme une succession ordonnée de phases, chacune comportant des opérations spécifiques à effectuer.
a) La phase de construction de l'objet
b) La formulation d'hypothèses
c) La détermination d'un cadre méthodologique
d) La délitation du terrain de recherche
e) Le recueil des données
f) L'interprétation des résultats

Ce modèle ordonné de la recherche en six phases offre à l'usage plusieurs intérêts sur le plan didactique : d'une part, il détermine des passages obligés : construire un objet, formuler des hypothèses, se doter d'un cadre méthodologique... Ces contraintes permettent d'éviter les biais classiques liés à l'attrait pour le terrain, le descriptif, l'événementiel. D’autre part, il favorise une mise à distance de la pratique et du vécu professionnel 
par le jeu discursif de la détermination d'un objet et de l'énonciation d'hypothèses communicables à des tiers extérieurs autres travailleurs sociaux, professionnels d'autres secteurs, formateurs, enseignants universitaires. Enfin, le processus ainsi décrit donne une représentation, à la fois globale et structurée dans le temps, des activités de recherche.

En dépit de ses qualités didactiques, ce modèle linéaire est luiaussi critiquable. En effet, la relative unité de chaque phase peut conduire à les comprendre comme indépendantes les unes des autres. Les défauts rencontrés proviennent non d'une erreur de raisonnement au sein d'une phase particulière, mais plutôt d'une articulation défectueuse entre 2 étapes.

L'exemple le plus révélateur est celui de la liaison entre les hypothèses et les méthodes de recueil des données. Souvent les hypothèses ne sont pas opérationnalisées, autrement dit éprouvables. Elles sont cohérentes par rapport à l'objet, mais demeurent très générales. Par conséquent, le questionnaire ou la grille d'observation est conçu indépendamment des hypothèses comme si elles n'existaient plus. Les étudiants apprentis chercheurs réintroduisent alors, dans l'élaboration de leur outil, leurs préjugés, leur expérience vécue, leurs impressions. La phase de formulation des hypothèses n'a servi à rien. D'autres dysfonctionnements liés à l'absence d'interactions entre une étape ou une autre sont observables : Plus en amont, les hypothèses ne recoupent pas l'objet défini ou s'en éloignent pour traiter d'autres thèmes. Plus en aval, le cadre méthodologique est difficilement compatible avec le terrain choisi ou la population impliquée.

En matière de recherche, la démarche n'est jamais linéaire avec progression d'un point initial jusqu'à son point d'achèvement ; c'est une démarche circulaire ou mieux en spirale avec retours fréquents en arrière. De tels retours peuvent parfois décourager l'étudiant comme le chercheur plus avéré. Ils sont pourtant indispensables, car en tant que démarche la méthodologie se veut une pratique : cette dernière n'a rien à voir avec l'enchaînement logique des étapes. Elle entend d'abord gérer l'incertitude et la complexité. 


\section{Le modèle cybernétique de la recherche comme boîte noire}

Nous venons de voir qu'une présentation linéaire de la recherche est insuffisante pour assurer la conduite d'une recherche. Si les différentes phases agissent et rétro-agissent les unes sur les autres, la recherche apparaît alors régie par un principe de totalité qui implique une cohérence d'ensemble. Il est facile de montrer qu'une modification dans l'une des 6 phases de la recherche est susceptible d'entraîner un changement affectant la totalité du processus.

\section{Exemple :}

une recherche est construite jusqu'à la phase de recueil des données et l'étudiant s'aperçoit au cours d'une pré-enquête auprès d'un échantillon que les salariés n'ont pas de demande explicitable par rapport au service ressources humaines de l'entreprise alors que toute la stratégie de l'enquête fonctionne sur cette notion. Plusieurs possibilités existent : soit il s'agit d'un simple problème de langage ou de formulation qui ne nécessitera qu'un aménagement technique. Soit les hypothèses traduisent les théories implicites des étudiants, il faut alors refaire un travail de définition des concepts utilisés (la demande), repenser l'objet d'étude puis formuler de nouvelles hypothèses. Dans la pratique, la nécessité de revoir des acquis théoriques et méthodologiques semblant pourtant définitifs est difficile à accepter (résistance au changement, baisse de motivation, critiques des normes universitaires...).

\section{Le modèle cybernétique amène à s'interroger sur les entrées et les sorties du processus de recherche :}

- Au départ, plusieurs types d'objectifs peuvent entrer dans un travail de recherche (affectifs, institutionnels, idéologiques...). Il importe donc d'opérer une explicitation minimale. Cela ne signifie pas qu'une recherche ne doive pas être investie affectivement, mais que seules des finalités objectivées et rationalisées 
peuvent entrer en ligne de compte (il s'agit en quelque sorte d'extraire le "venin » qui habite le chercheur). Chez les étudiants, la tendance à énoncer des objectifs sur le mode "j'ai envie de travailler sur... » ou «j'aimerais bien montrer que... » est souvent très forte. Dans les cas où elle prédomine, elle conduit à des difficultés importantes.

- En sortie, figurent évidemment les résultats de la recherche sous forme de rapports, mais il y a aussi des effets inattendus, pervers ou attendus : de nouvelles hypothèses ; la sensibilisation d'une population à un problème donné ; l'émergence d'une demande sociale par rapport à un équipement ; la formation de l'équipe de recherche, etc.

- L'analyse de ce qui sort (l'évaluation) n'est pas un exercice de style. Dans l'enseignement de la recherche, l'objectif n'est pas de réduire l'étude à la manipulation de séries statistiques, mais d'amener les étudiants futurs praticiens à ne formuler que des objectifs pouvant être évalués sans équivoque. De ce point de vue la recherche participe de l'effort de transparence dans les projets revendiqués souvent par les responsables et gestionnaires de terrain. Elle met en jeu la capacité des futurs professionnels à utiliser un langage moins idéaliste ou intuitif. La recherche dans l'effort d'explicitation qu'il suppose constitue donc un vecteur de la communication.

Sur un plan didactique, présenter la recherche comme globalement assimilable à un modèle cybernétique reste encore sommaire pour au moins trois raisons. Tout d'abord, il manque des phases intermédiaires : comment passe-t-on de l'objet aux hypothèses, des données collectées à leurs interprétations par exemple ? Ensuite, la nature des interactions entre les différentes étapes n'est pas explicitée, alors que l'essentiel n'est pas de savoir qu'il existe une interaction, mais de connaître comment elle fonctionne. Enfin, l'évaluation d'une recherche selon ce modèle s'opère à la fin alors que les régulations en relation avec les objectifs 
sont permanentes. Avec les conseillers de mémoire, l'évaluation se fait tout au long de la démarche.

\section{Le modèle systémique de la recherche}

Contrairement à ce que pose le modèle cybernétique, il ne suffit pas d'indiquer que les différentes phases interagissent entre elles sans préciser le contenu des interactions (modèle de la boîte noire). En adoptant un modèle systémique de la recherche (Quivy \& Van Campenought, 2011), il faut préciser ce qui se joue entre les interactions et tenter de répondre à quelques questions laissées en suspend :

- Comment se construit l'objet ? : La construction dérive d'une réflexion qui s'appuie sur des références théoriques, des définitions scientifiques, des observations empiriques, des savoirs professionnels, des théories implicites et leur confrontation.

- Comment passe-t-on aux hypothèses ? On le fait à travers cette démarche : Admettre et faire sienne un certain nombre de définitions, énoncer les questions pour lesquelles une réponse est attendue et avancer des réponses provisoires à ces questions. Dans beaucoup de recherche d'étudiants, les hypothèses ne sont que des fausses hypothèses (logique d'association spontanée). Le risque c'est d'avoir ici : une interprétation qui tourne en rond faute d'un minimum de théorisation, des études qui fonctionnent sur des théories implicites, etc.

- Comment rendre éprouvables les hypothèses ? 2 possibilités existent : Soit en les relativisant par rapport à un groupe plus restreint, dans le temps ou dans l'espace (dimension monographique). Soit en établissant des hypothèses principales puis des hypothèses secondaires pour les rendre opératoires (arborescence). 
- Comment interpréter les données recueillies ? Beaucoup d'analyses et d'interprétation ne font que paraphraser les résultats bruts ( 32 familles sur 93 habitent dans un logement de plus de 5 pièces... »). Ce biais provient souvent d'une absence d'hypothèse ou de propositions insuffisamment opérationnalisées. L'interprétation des données peut être décomposée en 5 phases : catégorisation des données brutes au moyen de règles techniques, une visualisation des données (tableau, graphique, etc.), calcul d'indicateurs (statistiques par ex.), analyse des conséquences de la (non) validation des hypothèses opératoires, discussion sur la façon dont la réalité répond à la question posée.

Ainsi en méthodologie de la recherche, il n'y a pas qu'une seule porte d'entrée, mais plusieurs. C'est à chacun de choisir, en fonction de sa situation, la porte qui lui convient. Toutes les portes par des circuits différents doivent mener au même résultat final. Le chercheur qui se trompe de porte risque vite d'être conduit à une impasse. Les différents modèles qui précèdent entendent constituer autant de portes d'entrée, mais non exhaustives. En prenant l'entrée qui lui convient et en cherchant à approfondir tout ce qui est impliqué par cette entrée, l'étudiant de master se rendra vite compte qu'il va insensiblement aborder d'autres problèmes, être malgré lui orienté dans le labyrinthe de la recherche vers d'autres entrées qui alors vont lui livrer leur secret, alors qu'elles ne l'auraient pas fait au départ.

\section{Quel doit être le rôle du directeur de mémoire ?}

En France, la direction des mémoires universitaires sera pratiquement toujours assurée par un enseignant-chercheur doté d'un niveau de formation supérieur à celui du diplôme préparé par l'étudiant. Par exemple, pour les mémoires de recherche de Master, le directeur doit posséder au moins un diplôme de Doctorat. En Doctorat, le directeur de thèse 
doit posséder une Habilitation à diriger des Thèses (HDR). Il appartient aux étudiants de faire ce choix en fonction du thème retenu, de l'approche théorique et méthodologique envisagée.

\section{Les 4 rôles du directeur de mémoire}

Le directeur de recherche est pour l'étudiant qu'il accompagne autant un stimulant qu'un analyseur extérieur de la recherche en train de se constituer et de la relation plus ou moins ambivalente que cet étudiant entretient avec son objet d'étude. D’où la nécessité pour l'étudiant de savoir utiliser pour le mieux la présence du directeur de recherche. Classiquement, il est possible de repérer quatre principaux rôles :

- Un rôle de conseil et de guideur, qui consistera à apporter remarques, critiques et suggestions dans les approches méthodologiques et théoriques.

- Un rôle de contrôle et d'évaluation afin de veiller à la rigueur scientifique de la démarche.

- Un rôle d'accompagnateur consistant à susciter l'autonomie de l'étudiant, seul responsable de sa production.

- Un rôle de formateur par lequel le directeur de mémoire accompagne le stagiaire dans sa formation, et favorise les liens entre le travail de mémoire et les contenus de la formation.

\section{Choix du directeur de recherche et suivi du travail}

Après la prise de contact avec plusieurs personnes, le choix définitif du directeur doit se faire en fonction d'une affinité de celui-ci avec l'objet d'étude proposé. Ce n'est pas le directeur qui détermine l'objet d'étude, sinon le stagiaire perd son autonomie de pensée et d'action et effectue une recherche " copie conforme " de la volonté du directeur. Par 
contre, c'est au directeur d'indiquer en quoi la proposition qu'il reçoit l'intéresse ; s'il ne manifeste qu'un faible intérêt, le stagiaire devra s'abstenir de poursuivre avec lui.

\section{Le suivi du travail implique simultanément :}

- Des rencontres de discussion : 4 à 5 séances plus ou moins espacées sur l'année universitaire constituent un repère intéressant pour mener un travail de recherche à bonne fin. De plus, elles ménagent l'autonomie du chercheur : trop peu de rencontres le coupent de son directeur et de la fonction critique qu'il est en droit d'attendre de lui. Trop de rencontres instaurent un système de dépendance, à terme paralysant.

- Des productions écrites, notamment au départ de la recherche avec présentation du projet de recherche explicité en 2, 3 ou 4 pages. Il peut y avoir ensuite des productions intermédiaires. À chaque production, le directeur réagit par écrit en effectuant un certain nombre de remarques.

- Le texte définitif de la recherche est soumis pour lecture au directeur, soit par tranches successives, soit globalement. Ce n'est qu'une fois l'imprimatur ${ }^{2}$ donné par le directeur de recherche, imprimatur le cas échéant accompagné d'un certain nombre de remarques, que le stagiaire peut faire éditer la version définitive de sa recherche. Il doit toujours se soucier d'un accord explicite avant de se lancer dans cette édition.

2 L'imprimatur (littéralement : qu'il soit imprimé !) est à l'origine une autorisation officielle de publier, donnée par une autorité de l'Église catholique. Elle est décrite dans le droit canonique, et donnée par l'ordinaire du lieu (l'évêque) où le livre est imprimé. Elle a pour origine la bulle pontificale Inter sollicitudines de 1515. Par extension, l'imprimatur peut désigner l'autorisation officielle de déposer le mémoire pour soutenance, donnée par le directeur de recherche à l'étudiant. 
Un contrat tacite au terme duquel sont définis l'étendue de l'étude, la méthodologie utilisée, le niveau d'investigation souhaité, l'ampleur quantitative du travail. Tout au long du processus, le conseiller de recherche vise à :

- Susciter l'autonomie de l'étudiant-chercheur seul responsable de sa production : ne pas inhiber l'initiative de l'étudiant en faisant des critiques incessantes.

- Adopter un compromis viable entre une trop grande dépendance et une trop grande indépendance de l'apprenti-chercheur.

- Manifester un intérêt curieux pour l'objet d'étude choisi : ne pas regarder le travail réalisé de trop loin.

- Mettre en évidence lors de la soutenance du travail les aspects positifs de la démarche, les progrès réalisés en cours de route; signaler les aspects lacunaires, mais sans demander au chercheur de se justifier; il l'aura fait en principe au cours des séances de discussion. Ce sera justement aux autres membres du jury de soutenance de demander des justifications, d’insister le cas échéant sur les aspects lacunaires du travail.

En acceptant de superviser un travail de recherche, le directeur s'engage en quelque sorte à être le promoteur de la recherche, bien entendu dans les limites de qualité.

\section{L'évaluation du travail}

Dans la direction d'un mémoire d'étudiant, la fonction d'évaluateur lors de la soutenance du mémoire est le deuxième rôle à assumer. Il peut changer, pour certains enseignants universitaires, le sens donné à la mission initiale. Evaluer, c'est mesurer les écarts existants entre les objectifs, le but à atteindre et la réalité concrète. Évaluer un mémoire, c'est rechercher si l'étudiant est arrivé à exposer l'idée qu'il a choisi d'examiner. C'est mesurer dans une échelle de valeurs si l'auteur a su poser une 
problématique et effectuer des recherches adéquates pour vérifier une hypothèse, s'il répond aux objectifs du mémoire.

Evaluation et validation qui interviennent au terme du processus de recherche sont pour certains conseillers bien séparées, pour d'autres télescopées, ce qui rend délicate la procédure de contrôle. À ce sujet, nous ferons trois réflexions :

Primo, du début à la fin de son accompagnement, le directeur de mémoire est à la fois guide, accompagnateur et évaluateur. La mission d'évaluateur peut paraître ponctuelle, elle est définie dans le temps puisque le directeur reçoit le mémoire terminé à une date précise et que la soutenance se déroule elle-aussi à une date précise dans un temps déterminé. Au moment où le directeur de mémoire reçoit le travail à corriger, il perd son statut de guide pour prendre celui d'évaluateur. C'est le passage d'un état à un autre qui est difficile parce que si concrètement, la transition s'effectue au moment de l'acte d'évaluation, la personne qui accepte de diriger un étudiant peut osciller sans arrêt entre ses deux missions au cours de l'élaboration du travail. Quand est-on directeur, quand eston évaluateur? Jusqu'où aller dans la direction, dans la guidance, dans l'accompagnement ? Comment en tant qu'enseignant peut-on évaluer un travail que l'on a dirigé, que l'on a guidé, que l'on a accompagné ? En fait, l'évaluation continue est déjà à l'œuvre à travers les différentes remarques et observations du directeur de recherche au cours du travail. L'évaluation est à la fois sommative et formative. Dans le cadre d'une formation qui vise le développement de compétences de chercheur, les données recueillies lors des activités d'accompagnement (évaluation formative) servent également à l'évaluation sommative. Durant toute la démarche de recherche, l'enseignant recueille, analyse et interprète des données sur l'apprentissage de l'élève. L'évaluation est donc continue bien qu'à la fin, elle prenne une allure plus formelle et exige le recours à des activités plus instrumentées et plus collective au sein du jury de soutenance. Tout le temps que dure la formation dans un cours, l'évaluation a une double fonction : elle est formative parce qu'elle vise à favoriser le développement de l'étudiant en l'informant sur ses apprentissages et en 
le guidant dans la conduite de son apprentissage ; elle est sommative en ce qu'elle contribue à la certification finale de l'intégration des apprentissages ou de la (des) compétence(s) grâce à l'information qu'elle fournit sur le développement du savoir-penser, du savoir-agir et du savoir-devenir de l'étudiant, sur leur combinaison et sur leur mobilisation.

Secundo, la validation intervient dès que le directeur de recherche donne l'accord d'imprimatur. Elle se poursuit notamment lors de la soutenance orale et de la délibération qui suit. En principe, la validation correspond à une courbe en " $J$ » : tout étudiant qui arrive au terme de son travail devrait être validé, car cette validation porte autant sur une capacité à maîtriser une démarche de recherche que sur certaines caractéristiques attachées au produit fini. S'il y a sélection, elle se fait en cours de route à travers le nombre des étudiants qui abandonnent. L'échec dans la validation peut néanmoins se rencontrer même si ce cas de figure devrait revêtir un caractère très exceptionnel. Dans ce cas, un usage fréquent consiste à demander à l'étudiant d'effectuer pour la session suivante une note complémentaire pouvant comporter selon les situations un recueil additif de données, un aperçu critique du premier travail réalisé, des propositions méthodologiques, etc.

Tertio, si la validation s'assure que les critères minimaux ont été respectés et permettent de donner à la recherche produite une reconnaissance sociale, l'évaluation terminale lors de la soutenance orale et de la délibération subséquente apprécie généralement deux principaux aspects : d'une part, la cohérence interne de la démarche (cadre méthodologique choisi, importance de l'investigation empirique, niveau de théorisation, maîtrise de concepts utilisés, etc.). D’autre part, est également appréciée la pertinence externe de l'étude (actualité et enjeux, voire originalité de l'objet d'étude retenu, intérêt des résultats vu le contexte actuel, prolongements possibles d'une telle étude et applications concrètes, etc.).

L'évaluation, lorsqu'elle séparable de la validation n'est pas encore, même à ce moment-là, chose simple. Elle met en jeu simultanément et de façon plus ou moins confuse : un jugement porte sur le produit, un 
jugement porte sur l'étudiant, un jugement porte sur le directeur-conseiller-promoteur de recherche.

\section{Principales difficultés et les pièges à éviter}

Pour terminer ce rapide tour d'horizon, nous voudrions tirer quelques leçons de notre expérience de formation à la méthodologie de la recherche en Université. Pour cela, nous commencerons par identifier les principales difficultés rencontrées par les étudiants, mais également par leurs conseillers de recherche.

\section{Les 9 péchés capitaux souvent rencontrés chez les étudiants}

On indiquera ici quelques-uns des pièges les plus caractéristiques dont est victime le chercheur novice, comme l'étudiant de Master. Éviter ces pièges c'est déjà lui donner des chances de mener à bien l'élaboration et la réalisation du projet de recherche.

- Absence de contact avec le directeur de recherche : Les contacts trop fréquents constituent bien entendu le piège opposé, mais ce cas de figure se rencontre moins souvent. Tout projet à commencer par le projet de recherche n'advient qu'à travers la relation. C'est cette relation qui permet le contact avec le directeur.

- Absence de terrain d'observation ou d'investigation : Le projet de recherche est clair et précis, mais on ne trouve pas l'organisation, la population, le milieu social susceptibles de se prêter à l'étude ; c'est le cas même de l'étude "abstraite".

- Absence de méthode définie pour appréhender l'objet d'étude : L'étudiant voit bien ce qu'il veut étudier, mais ne sait pas comment, par quel moyen s'y prendre. L'objet est alors insuffisamment construit ou trop abstrait. 
- Absence d'échéances temporelles : Tout est laissé à l'improvisation, sans fixation de contraintes, de temps qui servent de butoir. Une telle absence d'échéance peut renvoyer à une incapacité à gérer le temps. La question des hors délais se posent aussi assez souvent lorsque le travail escompté n'est pas réalisé dans les délais prévus. Dans ce cas, un temps supplémentaire limité peut être accordé et négocié avec le directeur de recherche. Parfois, lorsque l'étudiant veut un délai plus long, il vaut mieux pour lui changer de directeur de recherche et reprendre les choses à zéro.

- Incapacité de passer de l’objet réel à l'objet construit : Le projet en reste au sens commun, aux notions générales, voire aux prénotions qui paralysent. Le mieux dans ce cas serait de changer d'objet pour éviter l'impasse.

- Trop grande extension de l'objet construit : Le chercheur veut trop en faire ou mésestime la trop grande généralité de l'objet défini ; il est condamné rapidement à se laisser déborder par son objet d'étude.

- Formulation abstraite des différents paramètres du projet de recherche: Il s'agit d'une belle construction, bien précise, mais l'étude ne montre pas quel rapport elle entend avoir avec la réalité concrète, comment elle peut interroger cette réalité. Elle pèche par sa trop faible pertinence.

- Absence de recueil de données : Le projet n'implique pas de recueil de données, de recours à une quelconque investigation empirique ; le cas échéant le projet veut utiliser des données de seconde main, ou prendre des données recueillies antérieurement dans un autre contexte.

- Impossibilité à écrire : Le jeune chercheur se trouve dans un état d'incapacité à préciser sur le papier à espaces réguliers. L'état d'avancement de son travail par de brèves notes de synthèse. Cette incapacité peut accompagner le chercheur jusqu'à la rédaction finale du travail. Une telle incapacité sera combattue par la mise en oeuvre régulière et impérative de courtes 
matérialisations écrites. À l'incapacité à écrire s'oppose la diarrhée verbale. Cette dernière, peu fréquente, excelle à cultiver l'approximation, l'à peu près.

Plus fondamentalement, la réalisation d'un travail de recherche en master universitaire comporte au moins deux questions particulièrement aiguës, qui méritent de notre point de vue une attention particulière.

La première concerne le rapport au savoir des étudiants français. En effet, en comparant empiriquement l'attitude d'étudiants français avec celle d'étudiants d'autres nationalités (en particulier canadienne et étatsunienne), nous constatons chez eux un rapport à la théorie généralement très (trop ?) respectueux. Or, ce respect vis-à-vis de la littérature ne les incite pas toujours à suffisamment oser remettre en cause ce qu'écrivent les auteurs. Pour comprendre cela, nous faisons l'hypothèse que ce rapport à la littérature peut être observé à la fois sous l'angle du rapport au savoir (au sens large, les savoirs, savoir-faire, savoir-être) et du rapport à l'autorité. Le rapport au savoir tout d'abord, peut être conçu soit comme un rapport au savoir élaboré par la société savante et qu'il convient de diffuser dans la société ; soit comme un rapport partagé d'une certaine façon par toute la société dont il faut mettre en relation les différents dépositaires ; soit comme un rapport de production personnelle du savoir par l'appropriation de constructions théoriques empruntées à la société savante pour favoriser les ruptures et les reconstructions dans l'univers personnel de la connaissance. Selon le type de rapport au savoir qui est privilégié, on voit dès lors émerger des significations différentes au savoir : savoir scientifique et livresque difficilement inébranlable, savoir personnel (ce que l'on sait, que l'on a expérimenté...), etc. Bref, il est sans doute utile avec les étudiants de master d'insister sur le fait que le savoir est toujours un savoir en construction pour éviter qu'ils leur accordent le statut d'un savoir immuable qu'ils n'oseraient critiquer. Sous l'angle du rapport à l'autorité, la déférence observée en France à l'égard des auteurs et des publications, se matérialise par exemple par la large place qu'on accorde généralement à la présentation de la littérature dans les thèses 
de doctorat et même dans les mémoires de master. L'anthropologue G. Hofstede (1987) a effectué des études sociologiques en milieu de travail auprès de nombreux pays. Il montre notamment que les Français ont une distance hiérarchique plus prononcée que la majorité des autres nationalités. La conception française de l'autorité repose en premier lieu sur le statut et la dépendance relationnelle. Sans être totalement iconoclaste, sans doute est-il utile d'apprendre à ces étudiants à se montrer davantage critiques à l'égard de la littérature.

Une deuxième question pédagogique concerne le travail d'écriture comme moyen d'auto-formation et de professionnalisation. L'écriture, dans les travaux de recherche des étudiants, est à la fois moyen et médium, aussi bien pour la communication que pour l'élaboration et le développement de la pensée. Les caractéristiques, la qualité de l'écriture devraient constituer une préoccupation centrale. Une question importante, en particulier pour les formateurs et les décideurs, est celle des effets de l'écriture en formation. En France, le débat sur la présence, la forme ou le moment d'un mémoire dans la formation initiale des enseignants n'est pas clos. Peut-on donner une validation empirique au postulat des effets formatifs des dispositifs d'écriture ? Il est difficile, plus encore que d'apporter des «preuves » d'effets de la formation sur les pratiques professionnelles objectives, d'en chercher les causes : comment désintriquer les influences de tel et tel aspects de l'itinéraire de formation d'un professionnel ? En revanche, les effets subjectivement ressentis par les formés ont fait l'objet d'enquêtes nombreuses, par entretiens et questionnaires, pendant le temps de formation et lors des premières années d'exercice. Comme l'écrivent J. Crinon et M. Guigue (2006), « Les recherches sur l'écriture en formation offrent un reflet des tensions qui traversent la formation : selon le moment du questionnement, ses modalités, les stagiaires interrogés, les résultats sont contrastés. Le mémoire n'échappe pas au discours de disqualification et au sentiment que les choses ne se font pas au moment opportun, ni dans une durée suffisante. Mais il peut aussi prendre sens dans une continuité et être vécu sans dramatisation ». Aux chercheurs de continuer à apporter les analyses qui éviteront de confondre évaluation 
et indice de satisfaction comme de croire que les potentialités d'un dispositif de formation en garantissent les effets.

\section{Principaux défis posés aux conseillers de mémoire}

Cet examen des questions posées par l'enseignement de la méthodologie de recherche en master ne serait pas complet si nous ne faisions également état des difficultés rencontrées par les enseignants universitaires eux-mêmes à l'occasion du suivi de ces mémoires de recherche. Ils constituent pour eux autant de défis à surmonter.

Premier défi : La formation à la recherche ne se réduit pas à la réalisation d'un mémoire en fin de cursus de master, elle constitue également un moment institué d'un processus plus vaste qui lui donne sens. Autrement dit, la recherche ce n'est pas seulement la recherche des étudiants, c'est aussi la manière dont les enseignants et les professionnels sur le terrain font vivre la recherche. À quoi bon former par la recherche si les praticiens se contentent d'un exercice professionnel ne sollicitant pas prise de distance réflexive et travail d'écriture ? De la même façon, au sein d'un programme de master, ce sont aussi les autres activités pédagogiques proposées aux étudiants qui participent à la formation à la recherche. Cela implique par exemple une pédagogie qui dépasse le niveau de la transmission, c'est à dire non centrée exclusivement sur le contenu des disciplines. La capacité à trouver et synthétiser des informations peut également utiliser des pédagogies de type "classe inversée » (en anglais : "flipped classroom »). Cela peut encore vouloir dire une mise en place d'instances qui conduisent l'étudiant à se questionner sur sa pratique de stage et à la conceptualiser (réflexion sur les stages, mise en place de dispositifs d'évaluation réellement formatifs, travaux dirigés sur des thèmes précis, etc.). Dans cette perspective, il serait utile que les professeurs puissent ouvrir leurs classes à leurs collègues, se créer des temps et des lieux partagés de réflexion et d'expérimentation pédagogiques, réinventer le métier en infusant un peu 
partout les valeurs de la recherche et de l'intelligence collective. Mais on s'en doute, à cette étape, une des difficultés se trouve dans l'organisation même de l'enseignement universitaire, calquée sur une bureaucratie professionnelle qui souvent en France valorise un certain " individualisme enseignant ", souvent invoqué par les enseignants dans l'expression " chacun a sa personnalité ». Ces logiques à la fois bureaucratiques et professionnelles s'avèrent peu compatibles avec une configuration de type projet qu'implique - d'après ce que nous avons observé - le travail collaboratif. Ces logiques organisationnelles exigent d'autres modes de coordination que celles instaurées dans le système universitaire autour de l'unité : une matière, une promotion, un professeur.

Second défi : Accompagner la recherche réalisée en master par des professionnels en reprise d'étude pose des questions spécifiques. Tout d'abord, l'étudiant en emploi est un professionnel, ce qui veut dire que sans oublier les valeurs inhérentes à sa condition d'homme ou de femme, il possède un vécu professionnel composé de connaissances pratiques et cognitives, mais aussi d'émotions liées ou développées par les situations de travail qu'il a côtoyées au cours de son métier. Ce vécu, cette expérience a nécessairement forgé des référentiels d'interprétations, des valeurs, même s'il n'est pas toujours capable de les dire, de les expliciter. C'est aussi une personne en formation, conséquence d'une décision réfléchie, d'une volonté en connaissance de tous les efforts qu'entraînent une formation à l'âge adulte et des moyens nécessaires engagés pour parvenir au but. Dans ce cas, il existe fréquemment un lien étroit entre la problématique personnelle et/ou professionnelle et le sujet du mémoire. À la différence de certains étudiants de formation initiale qui sont en quête d'un sujet de recherche, l'adulte en reprise d'étude pêche fréquemment pas excès inverse. Il y a une surabondance de questions, entremêlées les unes aux autres, et la difficulté pour le conseiller de recherche consistera souvent à faire accepter au professionnel chercheur de retreindre son objet d'étude en laissant de côté de nombreuses questions qui ne peuvent être toutes traitées. De même, 
si le praticien réflexif (Schön, 1993) excelle à formuler des questions de départ dotées d'une grande pertinence professionnelle, ses bases théoriques et méthodologiques nécessitent par contre d'être davantage actualisées que celle d'étudiants plus jeunes réalisant leur master immédiatement après un baccalauréat (licence française).

Troisième défi : La connaissance des références théoriques pertinentes, qui renvoient à ses propres activités de recherche. Comment prétendre accompagner un étudiant de master dans son travail de recherche sans être soi-même chercheur ? Conseiller un étudiant sur les auteurs à lire, sur les méthodes d'analyse à utiliser, sur le format de la publication exige des compétences techniques. Le directeur de recherche doit s'appuyer sur une expertise scientifique forte qu'il a approfondie dans le cadre de mémoires et de thèses sur des sujets spécifiques, mais il doit aussi montrer une excellente culture générale scientifique dans tous les domaines qui touchent de près ou de loin à son sujet d'étude. Ses bonnes connaissances des protocoles expérimentaux liés à son domaine de recherche scientifique lui permettront de donner des indications claires aux étudiants (manipulations et tests en laboratoire...) et/ou de réaliser lui-même les expérimentations. Un bon niveau d'anglais est également souvent nécessaire afin de pouvoir orienter l'étudiant sur des références bibliographiques pertinentes et de favoriser les échanges avec des universités ou des organismes de recherche étrangers. De la même façon, sur le plan des qualités personnelles, le directeur de recherche doit se montrer créatif afin de trouver en permanence de nouvelles pistes de recherche ou des solutions face à des difficultés expérimentales. Tenace et persévérant, ses recherches peuvent s'inscrire sur des cycles longs avant d'obtenir des signes positifs de progression ; il doit être rigoureux, afin de mener à bien et selon des méthodes scientifiques l'ensemble des protocoles d'expérimentation. Il sait travailler en équipe et fédérer un groupe autour des projets de recherche menés. C'est un bon communicant qui présente l'état d'avancement des recherches aussi bien en interne (comités scientifiques) 
qu'en externe (formations, colloques...). Il possède également de bonnes qualités de rédaction en français et en anglais, afin de publier ses travaux dans des ouvrages scientifiques internationaux, la langue de rédaction d'usage étant l'anglais.

Quatrième défi : L'équilibre entre guidance et accompagnement. La diversité des expressions utilisées pour désigner l'enseignant-chercheur qui accompagne l'étudiant dans la réalisation de son mémoire est intéressante à relever. Tantôt qualifié de directeur de recherche, de conseiller, de guideur, de tuteur, de conseiller de recherche, etc. Cette diversité des expressions illustre la variété des postures qui peuvent être adoptées, notamment du point de vue de la directivité. À l'extrême, on peut encore retrouver la pratique du mandarinat consistant pour un professeur d'université à exercer un pouvoir important ou excessif, dans le choix du sujet de recherche des étudiants qu'il dirige. Dans ce cas, la direction de mémoire ou la guidance comporte une dimension directive, voire plus autoritaire. Guider c'est montrer le chemin, c'est aussi remettre dans la bonne direction lorsqu'on s'égare. C'est donc exercer une influence sur la personne. Diriger c'est indiquer le chemin que l'on a choisi, c'est laisser des traces et des repères à suivre, c'est aussi refuser le chemin que l'étudiant voudrait prendre dans le contexte du mémoire et qui ne semble pas pour le directeur de mémoire mener au but. Selon une posture moins directive, l'accompagnement implique pour le conseiller de recherche et l'étudiant de faire un bout de chemin à faire ensemble (Boutinet, Denoyel, Pineau \& Robin, 2007). Le sens du mot " accompagner " signifie alors marcher à côté, voire légèrement en retrait, en laissant l'étudiant avancer à son rythme, sans le bousculer. Accompagner ne veut donc pas dire précéder, mais partager quelque chose (du pain au sens étymologique du terme). Si une personne accompagne, cela veut dire que c'est toujours l'autre, l'accompagnée qui a l'initiative du chemin. Alors la fonction de directeur de mémoire est-elle de l'ordre de la guidance ou de l'accompagnement ? Sans doute un peu des deux. Cette situation de travail nécessite 
pour l'enseignant-chercheur d'accompagner l'étudiant pour lui permettre de dérouiller son propre appareil théorique, le huiler pour un meilleur fonctionnement, de ne pas être submergé par une angoisse massive déstructurante, de voir ce dont il peut être capable au travers d'expériences concrètes positives. Le directeur de mémoire peut allier les missions de direction, de guidance et d'évaluation à condition de s'effacer complètement derrière ses paroles et ses actes, sans s'approprier la recherche de l'étudiant. Il doit dynamiser la réflexion et l'énergie de celui-ci.

En définitive, qu'il soit étudiant ou professionnel en reprise d'étude, l'apprenti chercheur est toujours un acteur qui dans le système d'élaboration du mémoire proposé par son directeur ou par l'institution, trouvera toujours une marge de liberté. De ce fait sa conduite, sa réaction ne sera jamais entièrement prévisible. Lorsque le travail demandé est une recherche individuelle sur un sujet d'intérêt professionnel, il s'agit bien de débuter par une question dotée de pertinence et d'une certaine actualité. Dans l'absolu, le point de départ appartient donc à l'étudiant ainsi que le but qu'il s'est fixé, même si celui-ci est souvent flou. Si nous avons le point de départ et le point d'arrivée, il reste le chemin intermédiaire qui n'est pas rectiligne et qui présente souvent de nombreux carrefours. Ce sont ces carrefours ou choix à prendre qui parfois interrogent. Le directeur qui dirige exerce une influence ou entraîne l'étudiant dans la voie qu'il a choisie. Par contre, le directeur accompagnateur attend que l'étudiant prenne le temps de choisir le chemin tout en offrant des alternatives pour l'aider. En fait, les deux options sont envisageables à condition que le directeur dans une démarche que nous qualifierions de psychologique s'efface complètement derrière ce qu'il dit ou fait en essayant de mobiliser l'énergie réflexive de l'étudiant que nous appellerons va-et-vient entre la réflexion, fruit de la pensée et de la personnalité, et les différents savoirs intégrés. 


\section{Références}

ANGERS, M. Initiation pratique à la méthodologie des Sciences Humaines. Québec : CEC, 2000 [1996].

BACHELARD, G. La philosophie du Non. Paris : PUF, 1940.

BACHELARD, G. La formation de l'esprit scientifique. Paris : Librairie philosophique J. Vrin, 1938.

BANDURA, A. Self-efficacy: The exercise of control. New York: Freeman, 1997.

BEAUD, S., \& WEBER, F. Guide de l'enquête de terrain. Paris : La Découverte, 2003.

BOLLINGER, D., \& HOFSTEDE, G. Les Différences culturelles dans le management : comment chaque pays gère-t-il ses hommes ? Paris : Ed. d'Organisation, 1987.

BOUTINET, J.-P., DENOYEL, N., PINEAU, G., ROBIN, J.Y., Penserl'accompagnement adulte, ruptures, transitions, rebonds. Paris : PUF, 2007.

CRINON, J., \& GUIGUE, M. Écriture et professionnalisation. Revue française de pédagogie, n¹56, p. 117-169, 2006.

CRINON, J. Le mémoire professionnel des enseignants, observatoire des pratiques et levier pour la formation. Bruxelles : L'Harmattan, 2003.

DEPELTEAU, F. La démarche d'une recherche en sciences humaines. Paris : De Boeck, 2010.

DEVEREUX, G. De l'angoisse à la méthode dans les sciences du comportement. Paris : Flammarion [1967 pour l'édition originale en anglais], 1980.

GOMEZ, F. Le mémoire professionnel : objet de recherche et outil de formation. Bruxelles : De Boeck, 2001.

JORRO, A. Professionnaliser le métier d'enseignant. Paris : ESF éditeur, 2002. 
PAILLE, P., \& MUCCHIELLI, A. L'analyse qualitative en sciences humaines et sociales. Paris : Armand Colin, 2003.

QUIVY, R., \& CAMPENHOUDT, L. Manuel de recherche en sciences sociales (4ème ed.). Paris: Dunod, 2011.

SCHÖN, D. A. Le praticien réflexif. À la recherche du savoir caché dans l'agir professionnel [traduit et adapté par J. Heynemand et D. Gagnon], Québec : Les éditions Logiques, 1993.

WEINBERG, A. Comment faire une recherche ? Sciences humaines, Paris, ${ }^{\circ} 11$, novembre 1991, p. 26, 1991.

Reçu: 02/06/17

Recebido : 02/06/17

Approuvé : 08/07/17

Aprovado : 08/07/17 